\title{
A COMBINATORIAL PROOF OF SCHUR'S 1926 PARTITION THEOREM
}

\author{
DAVID M. BRESSOUD
}

\begin{abstract}
One of the partition theorems published by I. J. Schur in 1926 is an extension of the Rogers-Ramanujan identities to partitions with minimal difference three. This theorem of Schur is proved here by establishing a one-to-one correspondence between the two types of partitions counted.
\end{abstract}

Many classical partition identities state that for each positive integer $n$ the partitions of $n$ with parts restricted to certain residue classes are equinumerous with the partitions of $n$ on which certain difference conditions are imposed. Most prominent among these are Euler's identity: partitions into odd parts are equinumerous with partitions into distinct parts; and the Rogers-Ramanujan identities: for $r=1$ or 2 , partitions into parts congruent to $\pm r(\bmod 5)$ are equinumerous with partitions into parts with minimal difference two and smallest part greater than or equal to $r$. In 1926, I. J. Schur discovered the partition theorem which is a natural extension [2].

SCHUR's THEOREM. Given any positive integer $n$, the partitions of $n$ into parts congruent to $\pm 1(\bmod 6)$ are equinumerous with the partitions of $n$ into parts with minimal difference three and difference at least six between multiples of three.

Many proofs of Euler's identity exist, including several which explicitly exhibit the correspondence between the two types of partitions counted (e.g., see [1, §19.4]). For the Rogers-Ramanujan identities, no such correspondence has been established. The purpose of this paper is to explicitly exhibit a one-to-one correspondence between the two types of partitions counted in Schur's Theorem. What makes the correspondence possible for Schur's identity and not for the RogersRamanujan identities is that Schur's identity is a special case of the following partition theorem in which the parts restricted to certain residue classes are distinct.

SChUR's TheOREM (FUll Generality). Given positive integers $r$ and $m$ such that $r<m / 2$, let $C_{r, m}(n)$ denote the numbers of partitions of $n$ into distinct parts congruent to $\pm r(\bmod m)$, and let $D_{r, m}(n)$ denote the number of partitions of $n$ into distinct parts congruent to $0, \pm r(\bmod m)$ with minimal difference $m$, minimal difference $2 m$ between multiples of $m$. Then $C_{r, m}(n)=D_{r, m}(n)$ for all $n$.

The special case given above is $r=1, m=3$. Clearly, $D_{1,3}(n)$ counts the partitions of $n$ with minimal difference three, minimal difference six between

Received by the editors October 20, 1978 and, in revised form, June 20, 1979.

AMS (MOS) subject classifications (1970). Primary 05A17, 10A45.

(c) 1980 American Mathematical Society 0002-9939/80/0000-0288/\$01.75 
multiples of three. To see that $C_{1,3}(n)$ also counts the partitions of $n$ into parts congruent to $\pm 1(\bmod 6)$, the reader is referred to the correspondence proof of Euler's identity given in Hardy and Wright [1, \$19.4]. Following the correspondence given there, a one-to-one correspondence between odd parts with no multiples of three (i.e. parts $\equiv \pm 1(\bmod 6))$ and distinct parts with no multiples of three (i.e. distinct parts $\equiv \pm 1(\bmod 3))$ is easily established.

The correspondence given below will be for Schur's Theorem in its full generality.

Definition. Given positive integers $r, m, n$ and $k$ such that $r<m / 2, n-$ $m k(k-1) / 2>r k$, an $r, m$ underlying partition for $n$ and $k$, or an underlying partition, is a partition of $n-m k(k-1) / 2$ into exactly $k$ parts, each of which is congruent to $0, \pm r(\bmod m)$, and such that multiples of $m$ are distinct. (Example: $4,6,6,11,20,40,45$ is a 1,5 underlying partition for 237 and 7.)

LEMMA 1. There is a one-to-one correspondence between $r, m$ underlying partitions for $n$ and $k$ and the partitions counted by $D_{r, m}(n)$ which have exactly $k$ parts.

Proof. Let $a_{1} \leqslant a_{2} \leqslant \cdots \leqslant a_{k}$ be an $r, m$ underlying partition for $n$ and $k$ and, for $1<i<k$, define $b_{i}=a_{i}+m(i-1)$. Then $b_{1}<b_{2}<\cdots<b_{k}$ is a partition counted by $D_{r, m}(n)$, and this correspondence is uniquely reversible. (Example: $4,6,6,11,20,40,45$ corresponds to $4,11,16,26,40,65,75$.)

DEFINITION. An ordering of the parts in an $r, m$ underlying partition for $n$ and $k$, say $\left(a_{1}, a_{2}, \ldots, a_{k}\right)$, is called an $\Omega$-ordering if the following inequalities are satisfied for any $i$ and $j, 1 \leqslant i<j \leqslant k$.

(1) $a_{i} \equiv a_{j} \equiv 0(\bmod m) \Rightarrow a_{i}<a_{j}$;

(2) $a_{i} \equiv \pm a_{j} \equiv \pm r(\bmod m) \Rightarrow a_{i} \leqslant a_{j}$;

(3)

$$
\left.\begin{array}{l}
a_{i} \equiv \pm r(\bmod m) \\
a_{j} \equiv 0(\bmod m)
\end{array}\right\} \Rightarrow a_{i}+m i<\left\lfloor\frac{a_{j}+m i}{2}\right\rfloor_{r}
$$

(4)

$$
\left.\begin{array}{l}
a_{i} \equiv 0(\bmod m) \\
a_{j} \equiv \pm r(\bmod m)
\end{array}\right\} \Rightarrow\left\lfloor\frac{a_{i}+m i}{2}\right\rfloor_{r}<a_{j}+m i
$$

or equivalently,

$$
\left\lceil\frac{a_{i}+m(i-1)}{2}\right\rceil_{r}<a_{j}+m i .
$$

In this definition, $\lfloor A\rfloor_{r}$ (resp. $\lceil A\rceil_{r}$ ) is the greatest integer less than or equal to $A$ (resp. least integer greater than or equal to $A)$ and congruent to $\pm r(\bmod m)$.

LEMMA 2. Every underlying partition has a unique $\Omega$-ordering.

Proof. Given an $r, m$ underlying partition for $n$ and $k$, let $b_{1}<b_{2}<\cdots b_{k^{\prime}}$ be the parts congruent to $\pm r(\bmod m)$ and let $c_{1}<c_{2}<\cdots<c_{k^{n}}$ be the parts divisible by $m$. If $\left(a_{1}, a_{2}, \ldots, a_{k}\right)$ is an $\Omega$-ordering of this partition, then $a_{1}$ equals 
either $b_{1}$ or $c_{1}$. By properties (3) and (4) of an $\Omega$-ordering, if $b_{1}+m<$ $\left\lfloor\left(c_{1}+m\right) / 2\right\rfloor_{r}$, then $a_{1}=b_{1}$. If $b_{1}+m>\left\lfloor\left(c_{1}+m\right) / 2\right\rfloor_{r}$, then $a_{1}=c_{1}$. Thus $a_{1}$ is uniquely determined.

We proceed inductively, and assume that $a_{1}, a_{2}, \ldots, a_{j-1}$ have been uniquely determined, and that we have used the parts $b_{1}, \ldots, b_{s-1}, c_{1}, \ldots, c_{t-1}$. Thus $a_{j}$ is either $b_{s}$ or $c_{t}$. Again by properties (3) and (4), if $b_{s}+m j<\left\lfloor\left(c_{t}+m j\right) / 2\right\rfloor_{r}$, then $a_{j}=b_{s}$. If $b_{s}+m j>\left\lfloor\left(c_{t}+m j\right) / 2\right\rfloor_{r}$, then $a_{j}=c_{t}$. Thus $a_{j}$ is uniquely determined, and Lemma 2 is proved. (Example: The $\Omega$-ordering of 4, 6, 6, 11, 20, 40, 45 is $4,20,6,6,40,45,11$.)

Definition. Given a partition, $a_{1}<a_{2}<\cdots<a_{p}$, counted by $C_{r, m}(n)$, we subdivide it, working from left to right, into blocks of at most two parts such that if $a_{j}+m>a_{j+1}$ and if (1) $j=1$, or (2) $a_{j}>a_{j-1}+m$, or (3) $a_{j-2}$ and $a_{j-1}$ share a block, then $a_{j}, a_{j+1}$ share a block. Otherwise, $a_{j}$ inhabits a block by itself. The order of the partition is the number of such blocks. (Example: The partition $4,11,14,16,21,29,31,34,36,41$ counted by $C_{1,5}(237)$ is blocked as follows $4|11,14| 16|21| 29,31|34,36| 41$. It has order 7.)

LEMMA 3. There is a one-to-one correspondence between $r, m$ underlying partitions for $n$ and $k$ and the partitions counted by $C_{r, m}(n)$ which have order $k$.

Proof. Let $\left(a_{1}, \ldots, a_{k}\right)$ be an $r, m$ underlying partition for $n$ and $k$ with the $\Omega$-ordering. Define a partition $b_{1}, \ldots, b_{k}$ of $n$ by setting $b_{i}=a_{i}+m(i-1)$ for $1<i<k$. Each $b_{i}$ which is divisible by $m$ is replaced by the pair $\left\lfloor b_{i} / 2\right\rfloor_{r},\left\lceil b_{i} / 2\right\rceil_{r}$. From property (3) of the $\Omega$-ordering, we have that if $i<j, b_{i} \equiv \pm r(\bmod m)$ and $b_{j} \equiv 0(\bmod m)$, then $b_{i}+m<\left\lfloor b_{j} / 2\right\rfloor_{r}$. From property (4), we have that if $i<j$, $b_{i} \equiv 0(\bmod m)$ and $b_{j} \equiv \pm r(\bmod m)$, then $\left\lceil b_{i} / 2\right\rceil_{r}<b_{j}$. Thus the resulting partition is one counted by $C_{r, m}(n)$ with order $k$. This procedure is uniquely reversed if parts sharing the same block are first added together, and then $m(i-1)$ is subtracted from the $i$ th part as read from the left. (Example: $4,20,6,6,40,45,11$ becomes $4,25,16,21,60,70,41$ which becomes $4|11,14| 16|21| 29,31|34,36| 41$.)

Lemmas 1 and 3, taken together, give the desired correspondence which establishes Schur's Theorem.

I wish to thank Dr. K. A. Post for suggestions which were helpful in clarifying this proof.

\section{REFERENCES}

1. G. H. Hardy and E. M. Wright, An introduction to the theory of numbers, 4th ed., Clarendon Press, Oxford, 1960.

2. I. J. Schur, Zur additiven Zahlentheorie, S.-B. Preuss. Akad. Wiss. Phys.-Math. K1., 1926, pp. 488-495. (Reprinted in I. Schur, Gesammelte Abhandlungen, vol. 3, Springer, Berlin, 1973, pp. 43-50.)

Department of Mathematics, Pennsylvania State University, University Park, Pennsylvania 16802

Current address: School of Mathematics, Institute for Advanced Study, Princeton, New Jersey 08540 